\title{
High-intensity interval training benefits patients with T2DM
}

Physical activity improves cardiovascular health in patients with type 2 diabetes mellitus (T2DM), but its effects on cardiac structure and function are unknown. In a new study published in Diabetologia, highintensity interval training (HIIT; brief episodes of vigorous exercise interspersed with periods of low activity or rest) is shown to improve cardiac structure and function in patients with T2DM.

Previous studies have identified changes in left ventricular structure and function in patients with T2DM that precede the development of heart disease, which is the main cause of morbidity and mortality in these patients. As HIIT produces a more robust cardiac response than moderate regular exercise, HIIT has the potential to improve cardiac structure and function in patients with T2DM. However, until now, the benefits of HIIT in patients with T2DM had not been determined.

The researchers, from Newcastle University, UK, conducted a randomized controlled trial in which 28 patients with T2DM (whose disease was controlled by metformin and/or diet) were randomly assigned to HIIT $(n=14)$ or standard care $(n=14)$

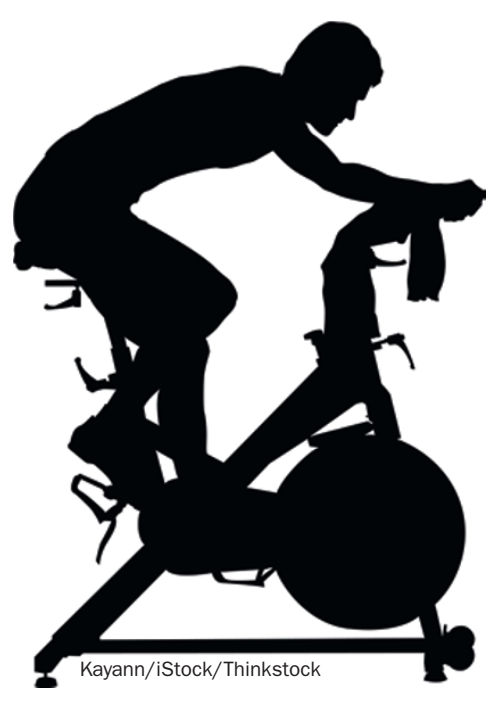

for 12 weeks. The HIIT group performed three cycle ergometry sessions per week, with training intensity based on the Borg rating of perceived exertion (RPE). Cardiac structure and function, levels of liver fat and glycaemic control were measured by $3.0 \mathrm{~T}$ MRI, ${ }^{1} \mathrm{H}$-magnetic resonance spectroscopy and oral glucose tolerance test, respectively.

Compared with standard care, HIIT induced structural and functional cardiac changes, with a $12 \%$ increase in left ventricular wall mass; systolic function (stroke volume) and end-diastolic blood volume were also increased. The exercise intervention also reduced levels of liver fat-a key pathogenetic factor in T2DM-by $39 \%$ and modestly reduced $\mathrm{HbA}_{1 \mathrm{c}}$ levels compared with standard care, even though HIIT had no effect on fasting glucose or insulin levels.

The authors acknowledge several limitations in their study, notably the inability of the MRI techniques used to identify the mechanisms responsible for the cardiac adaptations, the use of RPE as a proxy for training intensity (possibly less accurate than an objective measure), and the lack of dietary monitoring during the study.

"Although the direct benefits of HIIT to glycaemic control remain uncertain, HIIT is a potential therapy to moderate cardiac risk and reduce liver fat in type 2 diabetes [mellitus] and should be considered by clinicians alongside other regimens to improve glycaemic control," conclude the authors in their report.

\section{David Holmes}

Original article Cassidy, S. et al. High intensity intermittent exercise improves cardiac structure and function and reduces liver fat in patients with type 2 diabetes: a randomised controlled trial. Diabetologia doi:10.1007/s00125-0153741-2 